\title{
Scholarly Paper Impact
}

Xiaomei Bai, Fuli Zhang, Jie Hou, Ivan Lee, Xiangjie Kong, Amr Tolba, Feng Xia

\section{Source}

Xiaomei Bai, Fuli Zhang, Jie Hou, Ivan Lee, Xiangjie Kong, Amr Tolba. (2018). Quantifying the impact of scholarly papers based on higher-order weighted citations. PLOS ONE, vol. $13(3), e 0193192$.

Based on the observation that citations are inversely related to the geographical distance following an exponential distribution, the impact of each scholarly paper is defined as its average higher-order weighted quantum PageRank value:

$S\left(P_{i}\right)=\left\langle P_{i, m}\right\rangle:={ }^{\frac{1}{M}} \sum_{m=1}^{M} P_{i, m}$

where $S\left(P_{j}\right)$ represents the prestige score of a scholarly paper, $\square P_{i, m}$ represents the average value of higher-order weighted quantum PageRank scores, $M$ represents the iteration number of the algorithm, and $\mathrm{P}_{i, m}$ indicates the $m$-th value of higher-order weighted quantum PageRank scores. The concept of the prestige score is inherited from Quantum Google algorithm ${ }^{1}$, with the importance of a node corresponds to the prestige score of a scholarly paper in our work.

1 Paparo GD, Müller M, Comellas F, Martin-Delgado MA. Quantum Google algorithm. The European Physical Journal Plus. 2014;129(7):1-16. 